\title{
OPEN Evolutionary transition of doublesex regulation from sex-specific splicing to male-specific transcription in termites
}

\author{
Satoshi Miyazaki ${ }^{1,10 凶}$, Kokuto Fujiwara ${ }^{2,10}$, Keima Kai ${ }^{2}$, Yudai Masuoka ${ }^{2,3}$, Hiroki Gotoh ${ }^{4}$, \\ Teruyuki Niimi ${ }^{5,6}$, Yoshinobu Hayashi ${ }^{7}$, Shuji Shigenobu ${ }^{6,8}$ \& Kiyoto Maekawa ${ }^{9 凶}$
}

The sex determination gene doublesex ( $d s x$ ) encodes a transcription factor with two domains, oligomerization domain 1 (OD1) and OD2, and is present throughout insects. Sex-specific Dsx splicing isoforms regulate the transcription of target genes and trigger sex differentiation in all Holometabola examined to date. However, in some hemimetabolous insects, $d s x$ is not spliced sexually and its sequence is less conserved. Here, to elucidate evolutionary changes in $d s x$ in domain organisation and regulation in termites, we searched genome and/or transcriptome databases for the $d s \times$ OD1 and OD2 in seven termite species and their sister group (Cryptocercus woodroaches). Molecular phylogenetic and synteny analyses identified OD1 sequences of termites and C. punctulatus that clustered with $d s x$ of Holometabola and regarded them as $d s x$ orthologues. The Cryptocercus $d s x$ orthologue containing OD2 was spliced sexually, as previously shown in other insects. However, OD2 was not found in all termite $d s x$ orthologues. These orthologues were encoded by a single exon in three termites for which genome information is available; they were not alternatively spliced but transcribed in a male-specific manner in two examined species. Evolution of $d s x$ regulation from sex-specific splicing to malespecific transcription may have occurred at an early stage of social evolution in termites.

In insects, sex determination is cell-autonomously controlled by a cascade composed of several genes, where the doublesex $(d s x)$ encoding sex-specific transcription factors is located at the base ${ }^{1}$. In the cascade of the fruit fly (Drosophila melanogaster), the number of X chromosomes is the primary signal for sex determination ${ }^{2}$ and is sequentially transduced into the sex-specific splicing of Sex-lethal, transformer, and dsx, resulting in the sexspecific transcription of target genes responsible for their sex differentiation ${ }^{3,4}$. Although insect taxa differ in upstream genes encoding splicing regulators in this cascade, $d s x$ is constantly present among them as well as in some crustaceans and chelicerates that are non-insect arthropods $s^{1,5-7}$. Moreover, the $d s x$ orthologue is sexually spliced in all four major holometabolous insect orders examined to date (Coleoptera, Hymenoptera, Lepidoptera, and Diptera $)^{3,4}$; thus, such $d s x$ regulation is believed to be a general feature of the cascade in all insects. However, in hemimetabolous insects, $d s x$ is sexually spliced in two of four species recently examined, but is spliced alternatively and not sex-specifically in the others ${ }^{8,9}$. Furthermore, in some crustaceans and mites, $d s x$ does not produce sex-specific splicing isoforms and is instead expressed in a sex-specific manner; this controls their male

${ }^{1}$ Graduate School of Agriculture, Tamagawa University, Machida, Tokyo 194-8610, Japan. ${ }^{2}$ Graduate School of Science and Engineering, University of Toyama, Gofuku, Toyama 930-8555, Japan. ${ }^{3}$ Institute of Agrobiological Sciences, NARO (National Agriculture and Food Research Organization), Tsukuba, Ibaraki 305-8634, Japan. 'Department of Biological Science, Faculty of Science, Shizuoka University, Suruga-ku, Shizuoka 422-8529, Japan. ${ }^{5}$ Division of Evolutionary Developmental Biology, National Institute for Basic Biology, Okazaki, Aichi 444-8585, Japan. ${ }^{6}$ Department of Basic Biology, School of Life Science, The Graduate University for Advanced Studies, SOKENDAl, Okazaki, Aichi 444-8585, Japan. ${ }^{7}$ Department of Biology, Keio University, Yokohama, Kanagawa 223-8521, Japan. ${ }^{8}$ NIBB Research Core Facilities, National Institute for Basic Biology, Okazaki, Aichi 444-8585, Japan. ${ }^{9}$ Faculty of Science, Academic Assembly, University of Toyama, Gofuku, Toyama 930-8555, Japan. ${ }^{10}$ These authors contributed equally: Satoshi Miyazaki and Kokuto Fujiwara. ${ }^{\square}$ email: smiyazaki@agr.tamagawa.ac.jp; kmaekawa@sci.u-toyama.ac.jp 


\begin{tabular}{|c|c|c|c|c|c|}
\hline & Database & $d s x$ & Dmrt11 & Dmrt93 & Dmrt99 \\
\hline Zootermopsis nevadensis & Genome (Znev OGS v2.229) & No hit & scaffold668 & Znev05388 & Znev16235 \\
\hline Hodotermopsis sjostedti & $\begin{array}{l}\text { Transcriptome (DRA000538 and } \\
\text { DRA001044) }\end{array}$ & Hsjo_dsx (c35221, LC635719) & c18070 & c38968 & No hit \\
\hline Cryptotermes secundus & $\begin{array}{l}\text { Genome (Csec_1.0), Transcriptome } \\
\text { (PRJNA382129) }\end{array}$ & $\begin{array}{l}\text { Csec_dsx1 (XM_023861307), Csec_dsx2 } \\
\text { (XM_023858380) }\end{array}$ & XM0238633381 & XM0238639211 & XM0238500541 \\
\hline Reticulitermes speratus & $\begin{array}{l}\text { Genome (Rspe OGS1.0), transcriptome } \\
\text { (DRA010978) }\end{array}$ & $\begin{array}{l}\text { Rspe_dsx (scaffold6: 6307974..6309410, } \\
\text { LC635717) }\end{array}$ & RS007930 & RS006912 & RS002870 \\
\hline Coptotermes formosanus & Genome (CopFor1.0) & Cfor_dsx (scaffold506: 427884..429431) & GFG33987 & Scaffold9383 & GFG38119 \\
\hline Macrotermes natalensis & $\begin{array}{l}\text { Genome (Mnat_gene_vl.2.cds), transcrip- } \\
\text { tome (PRJNA382034) }\end{array}$ & Mnat_dsx (Mnat_08109) & No hit & Mnat_01812 & Mnat_08410 \\
\hline Nasutitermes takasagoensis & Transcriptome (DRA001046) & Ntak_dsx(G5ZWOJF02FLJ2Z ${ }^{\mathrm{a}}$, LC635718) & comp25194 & comp174542 & No hit \\
\hline Cryptocercus punctulatus & Transcriptome (PRJDB4695) & $\begin{array}{l}\text { Cpun_dsx } \boldsymbol{x}^{\mathrm{b}} \text { (Cpun_comp8195_c0_seq1, } \\
\text { LC635715, LC635716) }\end{array}$ & comp1991 & No hit & No hit \\
\hline
\end{tabular}

Table 1. Results of BLAST search for OD1 against the genomes and/or transcriptomes of seven termite species and their sister group (subsocial woodroach). Bold italic letters are gene names used in this study.

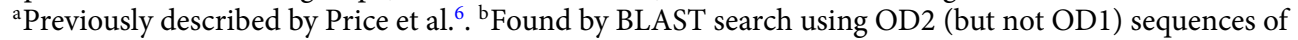
Blattella ds $x$ orthologues, followed by $5^{\prime}$ RACE.

differentiation ${ }^{5,7}$. Therefore, in contrast to knowledge based on holometabolous insects, the sex-specific regulation of $d s x$ may be evolutionarily labile among insects and non-insect arthropods and could have diversified in hemimetabolous insects.

A recent study by Price et $a .^{6}$ contributed a great deal of understanding to $d s x$ evolutionary history. They searched public databases of insect genomes and transcriptomes for $d s x$ homologues based on the presence of two domains conserved throughout holometabolous insects: the DNA-binding DM domain and the dimerization domain, referred to as oligomerization domain (OD) 1 and OD2, respectively. OD1 is found not only among $d s x$ but also doublesex/mab-3 related transcription factor (DMRT) genes that are present among metazoans and involved in their sexual development ${ }^{10,11}$, whereas OD2 is specific to $d s x^{12}$. They failed to obtain any evidence of the presence of $d s x$ in several hemimetabolous insects, including termites (infraorder Isoptera or epifamily Termitoidae), a monophyletic group within the cockroaches (Blattodea) ${ }^{13,14}$. In the lower termite Zootermopsis nevadensis, neither domain was found, and only OD1 was found in the higher termite Nasutitermes takasagoen$s i s^{6}$. A $d s x$ homologue has been identified in the genome of the German cockroach Blattella germanica (gene ID: PSN43312.1 ${ }^{15}$ ). Blattella $d s x$ was shown to contain both OD1 and OD2 (referred to as "DM domain" and "Dsx Dimerization domain", respectively, in Wexler et al. ${ }^{9}$ ) and to be alternatively spliced in a sex-specific manner as in holometabolous insects ${ }^{9}$. The rapid sequence divergence of $d s x$ (or part of it) might be the reason why the orthologues could not be found in termites. We hypothesise that the $d s x$ sequence (especially OD1 and/or OD2) diversified during termite evolution, and $d s x$ sequence diversification subsequently affected its regulation. These hypotheses can be examined using a comprehensive search of complete genome sequences and transcriptomic data in termites and in their sister group, subsocial woodroaches (Cryptocercus spp.) $)^{16-22}$.

The present study aims to elucidate evolutionary changes in $d s x$ in both domain organisation and its manner of regulation in the cockroach and termite clade. First, we exhaustively searched genome and transcriptome databases of the subsocial woodroach Cryptocercus punctulatus and seven termite species (Hodotermopsis sjostedti, Cryptotermes secundus, Reticulitermes speratus, Coptotermes formosanus, and Macrotermes natalensis, as well as Z. nevadensis and N. takasagoensis) for the OD1 and OD2 of $d s x$, and then examined whether the searched sequences were $d s x$ homologues based on molecular phylogeny and synteny. Second, we performed gene expression analysis based on quantitative RT-PCR and published transcriptome data, for one subsocial woodroach and two termite species. Third, to identify putative regulatory factors in the sex-specific transcription of termite $d s x$, transcription factor binding sites (TFBSs) were identified in the promoter region of the $d s x$ orthologue of $R$. speratus. The expression levels of putative transcription factors were compared between the sexes using published transcriptome data. Based on the results obtained, we conclude that $d s x$ regulation shifted from sex-specific splicing to male-specific transcription at an early stage of social evolution in termites.

\section{Results}

The search for doublesex orthologues in termites and a subsocial woodroach. We performed BLAST searches using the translated OD1 sequence of Blattella $d s x$ ( 45 amino acids) as a query against genome and/or transcriptome databases of seven termite species and one Cryptocercus woodroach species, after which two to four OD1-containing sequences were hit in each species (Table 1). BLAST searches were then performed using the translated OD2 sequence of Blattella $d s x$ ( 45 amino acids) as a query. A single sequence from the woodroach was hit (Cpun_comp8195_c0_seq1, Table 1), but no sequence was hit from any tested termites. Rapid amplification cDNA ends (RACE) PCR was performed using primers specific for Cpun_comp8195_c0_ seq1 (Table S1), and a single full-length transcript was obtained only from females. Then, using a reverse primer specific to the male-specific exon of Blattella dsx (located at terminal codon and 3'UTR, Table S1), another 3' end of coding sequence was amplified from males. As these transcripts contained an OD1 upstream of OD2 (Fig. 1), it was designated as $C$. punctulatus $d s x$ (Cpun_dsx). The amplified sequences downstream of OD2 were predicted to be the sex-specific exon(s) of Cpun_dsx. The determined nucleotide and putative amino acid sequences of the 


\section{subsocial woodroach}

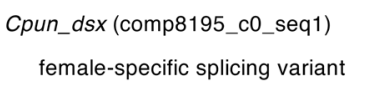

male-specific splicing variant

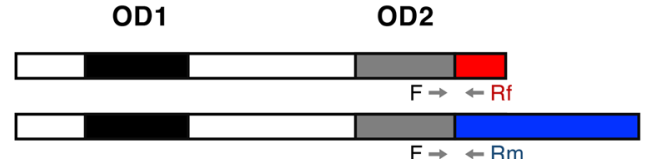

$\underline{\text { termites }}$

Hsjo_dsx (c35221)

Csec_dsx1 (XM_ 023861307)

Csec_dsx2 (XM_ 023858380)

Rspe_dsX

Cfor_dsx

Mnat_dsx (Mnat_08109)

Ntak_dsx (G5ZWOFJ02FLJ2Z)

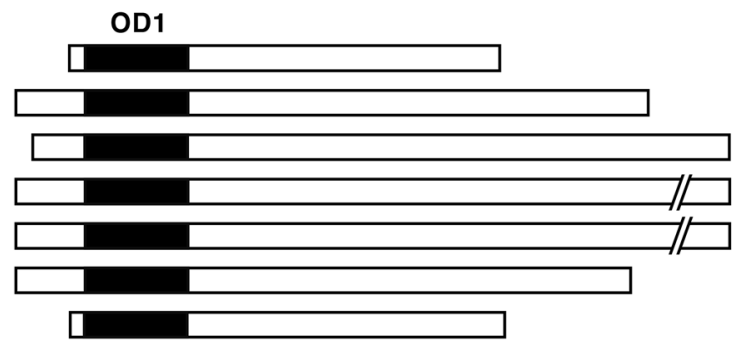

231 aа
$359 a a$
421 aa
$478 a a$
$516 a a$
$347 a a$
$239 a a$

Figure 1. Domain organisations of $d s x$ transcripts in a subsocial woodroach and in termites. Full-length coding sequence of $d s x$ transcripts in one woodroach and six termite species are shown as transcribed from left to right. The OD1 is shown as a filled box, whereas the sexually shared, female-specific, and male-specific OD2 is shown as a grey, red, and blue box, respectively. Numbers on the right of boxes are amino acid lengths of the translated $d s x$. Positions of primers for quantifying CPun_dsx expression by quantitative RT-PCR are shown in arrows: "F", "Rf", and "Rm" indicate the shared forward primer, reverse primer for the female-specific splicing variant, and reverse primer for the male-specific variant, respectively (Table S2).

Cpun_dsx splicing variants are available in the DDBJ/EMBL/GenBank databases (accession no. LC635715 and LC635716).

To examine the orthology of $d s x$ in insects, we performed a phylogenetic analysis using OD1 nucleotide sequences searched in termites and a Cryptocercus woodroach, as well as those of $d s x$ and Dmrt orthologues in other species. The phylogenetic analysis revealed that all $d s x$ reported in holometabolous insects, the German cockroach, and a water flea belonged to a single clade ( $d s x$ clade) (Fig. 2). This monophyletic $d s x$ clade also contained a single OD1 sequence from each of the four termites H. sjostedti (c35221 ${ }^{16}$ ), R. speratus (not annotated, scaffold 6 60: 6307974.0.6309410), Coptotermes formosanus (not annotated, scaffold 506 ${ }^{22}: 427884.0 .429431$ ), N. takasagoensis (G5ZWOJF02FLJ2Z ${ }^{16}$ ), and M. natalensis (Mnat_08109 ${ }^{18}$ ), and two sequences from Cryptotermes secundus (XM_023861307 and XM_023858380 ${ }^{15}$ ) (Fig. 2, Table 1). We confirmed that the sequences containing these $d s x$-clade OD1 did not possess a OD2 sequence that was unlike Cpun_dsx. No $d s x$-clade OD1 sequence from $Z$. nevadensis was identified in this phylogenetic analysis. The other OD1 sequences from insects formed clades specific to the DMRT11, DMRT93, and DMRT99 orthologues (Fig. 2).

To confirm whether the $d s x$-clade sequences in termites were $d s x$ orthologues, synteny analyses for $d s x$ were conducted based on the proximity of the $d s x$ and prospero (pros), which is well conserved among eight different holometabolous and hemimetabolous insect orders ${ }^{9,23}$. The gene encoding the $d s x$-clade sequence was located $83 \mathrm{~kb}, 29 \mathrm{~kb}$, and $80 \mathrm{~kb}$ from the pros orthologues (RS012493 ${ }^{24}$, GFG3663722, and MN005277 ${ }^{25}$ ) in scaffold 6 of R. speratus ${ }^{20}$, scaffold 506 of Coptotermes formosanus ${ }^{22}$ and scaffold 295 of M. natalensis (Mnat_08109), respectively. Therefore, these $d s x$-clade genes were designated as the termite orthologues of $d s x$ (Rspe_dsx, Cfor_dsx, and Mnat_dsx, Table 1). In C. secundus, both $d s x$-clade sequences, XM_023861307 and XM_023858380, and the pros orthologue (XM_023851644) were located in scaffold 829 (length: $88.5 \mathrm{~kb}$ ), 635 (length: $1.3 \mathrm{Mb}$ ), and 511 (length: 2.2 Mb), respectively. Because the scaffold lengths (especially for scaffold 829) appeared insufficient for the synteny analysis based on proximity (17-245 $\left.\mathrm{kb}^{9}\right)$, evidence of synteny in C. secundus could not be obtained. The synteny in $H$. sjostedti and $N$. takasagoensis could not be examined because their genome data are unavailable (as of 12 July 2021). Based on OD1 sequence similarity (Fig. 2), however, we designated these as $d s x$ orthologues (hereafter referred to as Csec_dsx1, Csec_dsx2,Hsjo_dsx, and Ntak_dsx, Table 1).

Splicing patterns and expression of doublesex homologues in termites and a subsocial woodroach. To confirm the sex-specific splicing of Cpun_dsx, expression levels of the predicted sexspecific splicing isoforms were quantified by RT-PCR using primers specific to the predicted isoforms (Fig. 1 and Table S2). The predicted female- and male-specific isoforms was confirmed to be abundantly expressed in females $(t=-6.68, p<0.01$, generalised linear model [GLM] $)$ and males $(t=7.88, p<0.01, \mathrm{GLM})$, respectively (Fig. 3), indicating that Cpun_ds $x$ was spliced in a sex-specific manner, as shown in holometabolous insects and the German cockroach B. germanica ${ }^{9,15}$. Next, to obtain the splicing isoforms of Hsjo_dsx, Rspe_dsx, and Ntak_ $d s x$, we performed $3^{\prime}$-RACE PCRs using gene-specific primers (Table S2) and amplified only the single fragment downstream of OD1 for each species (Fig. 1). The amplified downstream sequences of each species (accession no. LC635717-9) were consistent with those obtained by the aforementioned BLAST search. Although $d s x$ is composed of ca. five exons, of which two to three posterior exons are sexually spliced in almost all insects examined previously ${ }^{26}$, each of the predicted full-length transcripts of Rspe_dsx,Mnat_dsx,Csex_dsx1, and Csec_dsx2 was encoded by a single exon in their genome. Based on the transcriptome data in $R$. speratus ${ }^{21}, R s p e \_d s x$ was expressed only in male reproductives (primary kings), soldiers, and workers, but not in females, and in both 


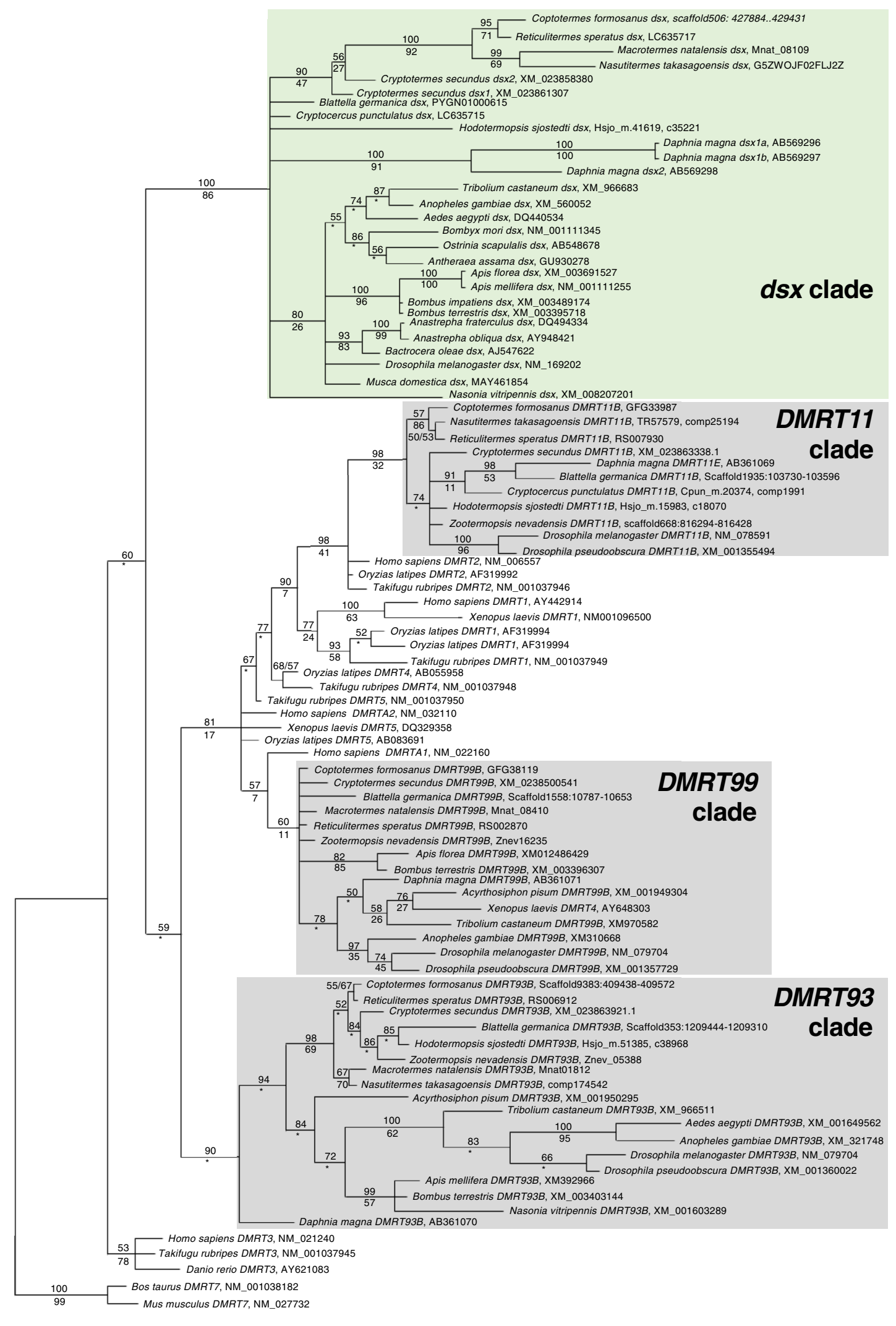

Figure 2. Molecular phylogenetic tree of $d s x$ and $D M R T$ homologues. Bayesian tree of $d s x$ and $D M R T$ of insects and crustaceans was constructed based on the OD1 sequences (135 bp with no gaps). Numbers shown above branches represent the Bayes posterior probabilities. Bootstrap values (1000 replicates) are shown below branches to indicate the level of support in the ML method. An asterisk indicates that a node is not supported by the ML method. 


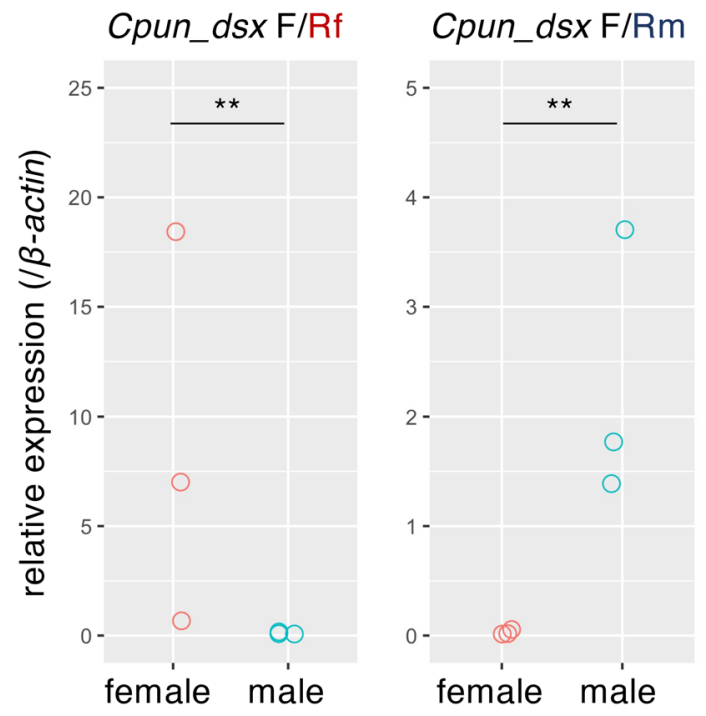

Figure 3. Expression patterns of Cpun_dsx in the female and male gonads of C. punctulatus adults. Left and right panels show the relative expression levels of its female- and male-specific isoform to those of $\beta$-actin, respectively. "F", "Rf", and "Rm" indicate the primers shown in Fig. 1. Replications of gonads are derived from individuals collected from three different kin groups. The expression levels were compared between sexes using GLM analyses. ${ }^{* *}$ indicates $p<0.01$.

heads $(t=3.99, p<0.01, \mathrm{GLM})$ and other body parts (referred to as just "body") $(t=10.97, p<0.001, \mathrm{GLM})$ (Fig. 4A). Quantitative RT-PCR also showed male-specific expression patterns even in the nymphs $(t=5.69$, $p<0.01$, GLM, Fig. 4B) and eggs $(t=3.10, p<0.05$, GLM, Fig. 4C). In addition, Ntak_ds $x$ was expressed only in males, regardless of caste $(t=3.70, p<0.01$, GLM, Fig. 4D). These results indicated that termite $d s x$ was not alternatively spliced, but transcribed in a male-specific manner.

Predicted binding sites and expression patterns of putative regulatory factors for male-specific transcription of Rspe_dsx. To examine the proximate mechanism for male-specific transcription of termite $d s x$, we searched for putative transcription factors that bind to the promoter region of Rspe_dsx, and those that are expressed in a male- or female-specific manner. First, $1 \mathrm{~kb}$ upstream of the transcription start site of $R s p e \_d s x$ was extracted as a transcriptional regulatory region, as defined by Toyota et al. ${ }^{27}$. Next, de novo motif discovery was performed for the promoter using hypergeometric optimisation of motif enrichment (HOMER) $\mathrm{v} 4.11^{28}$, based on the equipped motif library of known transcription factors in insects. Although 32 putative TFBSs were detected in the promoter, 20 homologues out of the 32 transcription factors existed in the Reticulitermes genome, of which five were duplicated in the genome (Table 2). Based on the transcriptome data in $R$. speratus $^{21}$, however, none of them were expressed in a sex-specific manner in either heads or bodies, except for the vielfaltig $(v f l)$ orthologue (GLM, Table 2). The expression levels of the $v f l$ orthologue were significantly different between sexes only in the bodies of reproductives, but similar patterns were not observed in other castes (Fig. S1).

\section{Discussion}

The $d s x$ orthologue of the subsocial Cryptocercus woodroach was spliced in a sex-specific manner, similar to that of the gregarious Blattella cockroach and holometabolous insects (Fig. 5). Surprisingly, however, termite $d s x$ homologues did not exhibit sex-specific splicing, and their transcription was regulated in a male-specific manner. Male- or female-biased expression of $d s x$ has been reported in at least 8 species of non-insect arthropods, including water fleas ${ }^{5,27}$, red claw crayfish ${ }^{29}$, Chinese shrimp ${ }^{30}$ (Crustacea), and mite ${ }^{7}$ (Chelicerata), suggesting that sex-biased $d s x$ transcriptions are an ancestral state in Arthropoda ${ }^{9}$. In contrast, $d s x$ produces sex-specific splice isoforms in major holometabolous insect orders (Coleoptera, Hymenoptera, Lepidoptera, and Diptera) and those hemimetabolous insects examined previously ${ }^{6,9,26}$ except for the louse Pediculus humanus and the hemipteran Bemisia tabaci. These two hemimetabolous insects have $d s x$ homologues with non-sex-specific isoforms $s^{8,9}$. Consequently, the sex-specific alternative splicing of $d s x$ would have been acquired early in the evolution of insects, and secondarily lost in some hemimetabolous insects ${ }^{9}$, including termites. Within the cockroach and termite clade, after ancestral termites diverged from the common ancestor of subsocial Cryptocercus and eusocial termites (around 140.6 MYA, ranging from 112.6 to $170.5 \mathrm{MYA}^{31,32}$ ) the data suggest that sex-specific $d s x$ splicing was lost, and the male-specific $d s x$ transcription was alternatively acquired in eusocial termites (Fig. 5). It remains unclear whether the $d s x$ orthologue was actually lost in the genome of $Z$. nevadensis. However, it is interesting to note that $Z$. nevadensis has a genome half the size of other termites $(562 \mathrm{Mb})^{19}$, suggesting the possibility that some genes may be missing. A further exhaustive search is needed. 
A Rspe_dsx

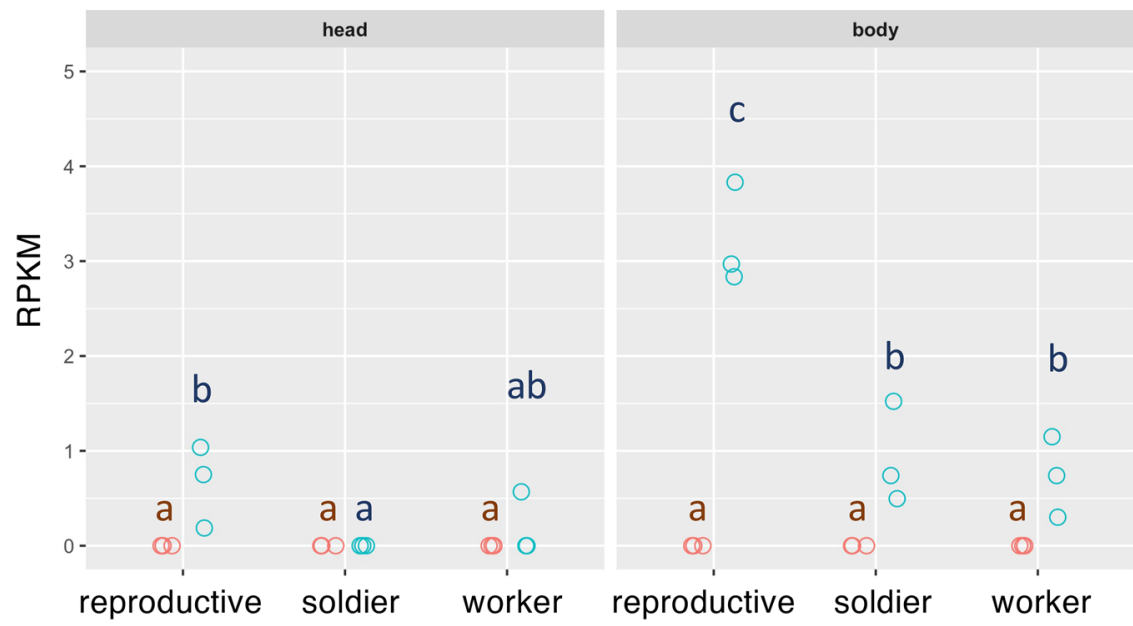

female

male
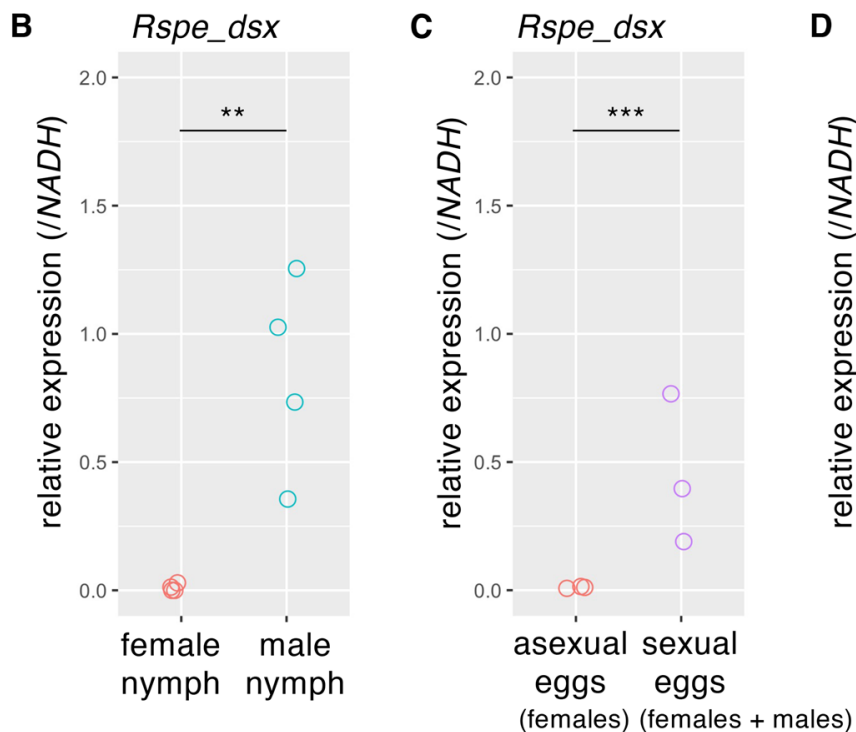

D Ntak dsx

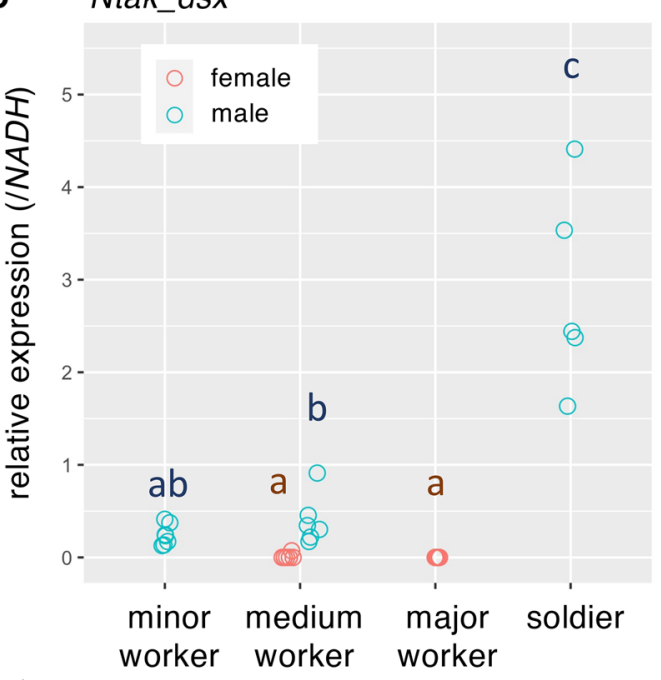

Figure 4. Sex-specific expression patterns of Rspe_dsx and Ntak_dsx. (A) Reads per kilo base per million mapped reads (RPKM) of Rspe_dsx in each sex and in each caste of $R$. speratus, which were re-analysed RNAseq data (DRA010978, biological triplicates ${ }^{21}$ ). (B) Rspe_dsx expression levels relative to those of NADH in female and male nymphs. All nymphs examined were collected from a single colony (i.e., siblings). (C) Relative Rspe_dsx expression in eggs produced asexually and sexually. The former are fated to develop into females, whereas the sexual fate of the latter was not determined. Total RNA was extracted from 30 eggs, and three RNA samples were derived from different colonies. (D) Ntak_dsx expression levels relative to those of NADH in each sex and in each caste of $N$. takasagoensis. All castes examined were collected from a single colony (i.e., siblings). In (A) and (D), the effects of sex, caste, and their interaction on gene expression levels were evaluated using GLM followed by multiple comparison using linear hypothesis testing with Tukey adjustment in the "multcomp" R package. Different letters indicate significant differences according to multiple comparison $(p<0.05)$. In (B) and $(\mathbf{C})$, relative expression levels of Rspe_ds $x$ were compared between sexes using GLM analyses. ${ }^{* *}$ and ${ }^{* * *}$ indicates $p<0.01$ and 0.001 in GLM, respectively.

Although $d s x$ consists of approximately five exons in 145 species belonging to 39 holometabolous insect families ${ }^{26}$, two $d s x$ paralogs ( $d s \times 1$ and $\left.d s \times 2\right)$ in water fleas consist of four and two exons, respectively ${ }^{5}$. Its homologue in Chinese shrimp consists of two exons $s^{30}$, and those in mites $^{7}$ and termites consist of only a single exon (Fig. 5). Moreover, the present study showed no homologous OD2 in termite $d s x$ (Fig. 5), suggesting that OD2 has indeed been lost or has diverged beyond our ability to detect it ${ }^{6}$. OD2 is conserved in $d s x$ orthologues with sex-specific splicing in holometabolous insects, as well as in those with sex-specific transcription in water fleas ${ }^{5,27}$ and Chinese shrimp ${ }^{30}$ but not in red claw crayfish ${ }^{29}$. Both OD1 and OD2 are related to binding target DNA as a dimeric DNA binding unit in the fruit fly D. melanogaster: OD1 enables Dsx to bind target DNA and dimerizes weakly in a DNA-dependent manner ${ }^{12}$, whereas OD2 is necessary for dimerization and enhances DNA recognition of OD $1^{33}$. In addition, fly OD2 contains sex-specific spliced sequences, which may be involved in the formation of sex-specific units for transcriptional regulation of downstream target genes through either its 


\begin{tabular}{|c|c|c|c|}
\hline Gene symbol & Gene ID in R. speratus & FDR $^{\mathrm{a}}$ in heads & FDR $^{a}$ in bodies ${ }^{b}$ \\
\hline $\operatorname{Trl}$ & RS014506 & 1.000 & 0.176 \\
\hline$g r h$ & RS004650 & 1.000 & 0.825 \\
\hline$C f 2-I I$ & RS004984 & 1.000 & 0.778 \\
\hline ara & RS009368 & 1.000 & 0.492 \\
\hline Hr46 & RS006489 & 1.000 & 0.910 \\
\hline$b r-Z 4$ & RS006084 & 1.000 & 0.970 \\
\hline$b r-Z 4$ & RS006083 & 1.000 & 0.628 \\
\hline$b r k$ & RS014094 & 1.000 & 0.217 \\
\hline$b r-Z 3$ & RS006084 & 1.000 & 0.970 \\
\hline$d v e$ & RS006834 & 1.000 & 0.836 \\
\hline$t t k$ & RS007158 & 1.000 & 0.914 \\
\hline$t t k$ & RS007159 & 1.000 & 0.931 \\
\hline pros & RS012493 & 1.000 & 0.890 \\
\hline pros & RS012492 & 1.000 & 0.217 \\
\hline$v f l$ & RS003882 & 1.000 & $0.275 \times 10^{-3}$ \\
\hline$a p$ & RS004033 & 1.000 & 0.913 \\
\hline$a p$ & RS004477 & 1.000 & 0.633 \\
\hline$D$ & RS010190 & 1.000 & 0.822 \\
\hline prd & RS008463 & 1.000 & 0.967 \\
\hline Hsf & RS003326 & 1.000 & 0.406 \\
\hline$s u(H w)$ & RS012357 & 1.000 & 0.791 \\
\hline$b r-Z 2$ & RS006084 & 1.000 & 0.970 \\
\hline$b r-Z 2$ & RS006083 & 1.000 & 0.628 \\
\hline Pnt & RS003359 & 1.000 & 0.807 \\
\hline$s d$ & RS008357 & 1.000 & 0.919 \\
\hline
\end{tabular}

Table 2. Transcription factors with putative binding sites detected in the R. speratus genome and their expression differences between sexes. ${ }^{a}$ FDR based on GLM with sex using edgeR. ${ }^{b}$ Body: the thorax and abdomen.

\begin{tabular}{|l|lcc}
\hline $\begin{array}{l}\text { Evolutionary changes in } d s x \\
-\quad \text { loss (or divergence) of } \mathrm{OD2} 2 \\
-\quad \text { unification of gene structure } \\
\text { - transition to male-specific } \\
\text { transcription }\end{array}$ \\
\hline $\begin{array}{l}\text { Evolution of eusociality } \\
\text { in termites }\left(\sim 134 \mathrm{MYA}^{32}\right)\end{array}$
\end{tabular}

Figure 5. Evolutionary changes in $d s x$ regulation associated with social evolution in termites. The presence of OD1 and OD2, numbers of exons containing coding sequences $\left(^{\dagger}\right)$, and regulatory manner of $d s x$ homologues were compared among the German cockroach, the subsocial woodroach, and seven termite species. "-" and "?" mean "not detected" and "unknown", respectively. ${ }^{*}$ Data on B. germanica were cited from Wexler et al. ${ }^{9}$. The tree topology was based on that of Bourguignon et al. ${ }^{57}$, Bucek et al. ${ }^{31}$, and Evangelista et al. ${ }^{32}$.

sex-specific interactions with the transcriptional machinery or its sex-specific DNA binding ${ }^{12}$. The decreasing numbers of $d s x$ exons in some arthropods and termites and the loss (or divergence) of OD2 in termites would be associated with the evolution of $d s x$ regulation, although it remains unknown whether such evolutionary changes in the domain and exon organisation were the cause or consequence of the loss of sex-specific splicing. Future studies that aim to characterize the $d s x$ orthologue in Mastotermes darwiniensis, the earliest branching termite lineage, might help clarify the issue. 
The sex determination cascade has never been examined in termites. Almost all orthologues of sex determination genes reported in holometabolous insects (e.g., transformer, transformer-2, and Sex-lethal) are conserved in the genome of R. speratus ${ }^{24}$. However, these orthologues encode splicing factors and are unlikely to directly regulate male-specific $d s x$ transcription. Male-specific transcription should be regulated by a transcription factor expressed in a sex-specific manner in every caste, as shown in termite $d s x$. Although we found 27 putative TFBSs in the Rspe_dsx promoter region, no orthologues of 27 transcription factors showed sex-specific expression patterns similar to those of $R s p e \_d s x$. However, our motif search was based on the binding motifs found in the fruit fly $D$. melanogaster. In addition, unidentified binding motifs could be present out of the transcriptional regulatory region examined in this study. Further investigations are needed to determine whether the unidentified transcription factors expressed in a sex-specific manner would regulate male-specific $d s x$ transcription in termites.

Although $d s x$ was sexually spliced in gregarious Blattella and subsocial Cryptocercus cockroaches, a single $d s x$ transcript was expressed only in the male of eusocial lower and higher termites. Given that cooperative brood care by parents and a generational overlap was likely acquired in the last common ancestor of Cryptocercus woodroach and termites ${ }^{32}$, the transition in $d s x$ regulation in termites is associated with the evolution of eusociality, especially the acquisition of reproductive division of labor (Fig. 5). Such association between $d s x$ expression and social traits also has been suggested in ants. The $d s x$ orthologues were sexually spliced in all 5 ants examined, whereas their $d s x$ was expressed in a male-biased manner in 3 species with an environmental caste determination system, but not in 2 species with a genetic caste determination system ${ }^{34}$. Additionally, the OD2 was present in 2 basal ant species, but absent in 17 ants belonging to the subfamilies with more advanced social traits ${ }^{34}$.

The male-specific transcription of termite $d s x$ would play a role in the regulation of reproductive division of labour, particularly in soldiers, the first sterile caste that evolved in Isoptera ${ }^{35}$. Some species, particularly in the crown termite family Termitidae, have sex-specific or sex-biased soldier ratios and soldier differentiation pathways $^{36}$. For example, all soldiers are females in most species of the subfamilies Termitinae and Macrotermitinae, whereas all soldiers are males in most of the examined species in Nasutitermitinae. Soldier differentiation requires high juvenile hormone titres in workers, and the strongly biased soldier-sex ratio may be rooted in differences in juvenile hormone titres (and probably related gene expression levels) between male and female workers $^{37,38}$. Reproductive caste differentiation is also regulated in a sex-specific manner (reviewed in Oguchi et al. ${ }^{39}$ ). "Queen genes" with high expression levels in female reproductives have been identified in some species (reviewed in $\mathrm{Korb}^{40}$ ), and in male reproductives upregulated genes likely associated with male fertility have been identified in species with available genome sequences ${ }^{15,19,24}$. Termite $d s x$ could regulate this sexually dimorphic gene expression by upregulating or downregulating the expression of its target genes in a male-specific manner, resulting in reproductive and non-reproductive division of labour.

\section{Materials and methods}

Insects. Seven mature colonies of R. speratus were collected in Toyama Prefecture in 2016 and 2019. One H. sjostedti mature colony was collected in Yakushima Island, Kagoshima Prefecture in 2015. Two N. takasagoensis mature colonies were found on Ishigakijima Island, Okinawa Prefecture, in 2017 and 2018. They were kept in the laboratory at ca. $25^{\circ} \mathrm{C}$ in constant darkness. Three kin groups of C. punctulatus were collected at Mountain Lake Biological Station, Giles County, VA in April 2015-2017 $7^{41}$ and kept at $15^{\circ} \mathrm{C}$ in constant darkness. Testes and ovaries were dissected from three males and three females, respectively, and stored at $-80{ }^{\circ} \mathrm{C}$ until RNA extraction for subcloning and expression analyses of CPun_dsx.

BLAST searches for $d s x$ and $d m r t$ homologues in termites and cockroaches. tBlastX searches were performed against genome and transcriptome databases of six termite species and one Cryptocercus woodroach species (Table 1), using SequenceServer ${ }^{42}$. Either the OD1 (45 amino acids) or OD2 (45 amino acids) sequences of $B$. germanica $d s x$ were used as queries. Sequences with an E value less than $10^{-5}$ were selected as candidates for $d s x$ orthologues and used for phylogenetic analysis.

Construction of phylogenetic tree of OD1 sequences and identification of $d s x$ orthologues. To identify the $d s x$ orthologue, a phylogenetic tree of OD1 sequences ( $135 \mathrm{bp}$ with no gaps) was constructed according to previous studies ${ }^{5,43,44}$. We used 84 OD1-containing genes (Table S3 'OTU'). The DMRT7 genes of vertebrates (mouse and cattle) were used as the outgroups. Phylogenetic relationships were inferred using Bayesian inference (BI) and maximum likelihood (ML) methods. For BI, the most appropriate model of sequence evolution was determined using the model selection option implemented in MEGA version $7.0 .21^{45}$, and the GTR + G model was selected. Using MrBayes version 3.2.6 $6^{46}$ a total of 100,000 trees were obtained (ngen $=10,000,000$, samplefreq $=100)$. The first $25 \%$ of these $(25,000)$ were discarded as burn-ins, and a $50 \%$-majority-rule consensus tree was produced. For ML, 1000 bootstrap replicates were performed based on the same model of sequence evolution as BI in MEGA 7.0.21, with the default tree inference options.

Synteny analysis. According to Wexler et al. ${ }^{9}$, the synteny of $d s x$ was examined based on the proximity to the transcription factor prospero (pros). The $d s x$ locates at the genomic position close to pros, with an intervening distance between 17 and $245 \mathrm{~kb}$ in eight different insect orders (Blattodea, Orthoptera, Ephemeroptera, Phthiraptera, Thysanoptera, Hymenoptera, Coleoptera, and Lepidoptera $)^{9,23}$. To identify the pros ortholog in each termite, tBlastX searches were performed against gene models of R. speratus ${ }^{24}$ and M. natalensis ${ }^{18}$ using SequenceServer ${ }^{42}$, and BlastX searches against predicted protein databases of Cryptotermes secundus ${ }^{15}$ and Coptotermes formosanus ${ }^{22}$. The pros sequence of B. germanica (Accession No. PYGN01000615) was used as a query. To show the genomic position, tBlastN searches then were performed against the genome database of Cryp- 
totermes secundus ${ }^{15}$ and Coptotermes formosanus ${ }^{22}$, querying with the obtained Pros protein sequence in each species using SequenceServer ${ }^{42}$.

Subcloning of the candidate $d s x$ orthologues. Female and male alate reproductives (winged adults) of $R$. speratus were obtained from three colonies collected in 2016. According to previous studies ${ }^{47,48}$, incipient colonies were established using unrelated female and male alates. A total of 30 eggs was obtained, and 3 primary queens and 3 primary kings were randomly selected from 20 colonies at 1.5 months after colony establishment. Total RNA of all the eggs was extracted and treated with DNase I using the ReliaPrep RNA Tissue Miniprep System (Promega, USA). Total RNA of the primary queens or kings was extracted from the body (except for the head parts, three individuals of each sex) using ISOGEN II (Nippongene, Japan). Total RNA of female or male alates of $N$. takasagoensis (using the colony collected in 2017) was extracted from the body (except for the head parts, five individuals in each sex) using ISOGEN II. Total RNA from the secondary queen or king of H. sjostedti (using the colony collected in 2015) was extracted from the body (except for the head part, one individual in each sex) using ISOGEN II. An ovary and testis of $C$. punctulatus were dissected from a female adult and male adult, respectively. Total RNA was extracted from each of the gonads using ISOGEN I and II, and then treated with RNase-free DNase I (Takara, Japan). The purity and quantity of the extracted RNA were measured using a NanoVue spectrophotometer (GE Healthcare Bio-Sciences, Japan).

The 5' and 3' ends of Cpun_ds $x$ were amplified using the SMARTer RACE 5'/3'kit (TaKaRa, Shiga, Japan) and gene-specific primers designed for OD2 (“Cpun_dsx OD2 5'RACE" and “Cpun_dsx OD2 3'RACE", Table S1). Its male-specific exon was amplified using Advantage 2 Polymerase Mix (TaKaRa), the gene-specific primer "CPun_dsx OD2 3'RACE", and a reverse primer in the male-specific exon of Blattella dsx, which was located at terminal codon and 3'UTR (“Cpun_dsx male-specific exon-R", Table S1). The 3' ends of Rspe_dsx, Hsjo_dsx, and Ntak_ds $x$ were also amplified using the SMARTer RACE $5^{\prime} / 3^{\prime}$ Kit and newly designed gene-specific primers (Table S1). The amplified fragments were subcloned into the pGEM-T easy vector system (Promega), and the nucleotide sequences of each fragment were determined using ABI Prism Big Dye Terminator v3.1 Cycle Sequencing Kit in conjunction with a 3500 Genetic Analyzer (Applied Biosystems, Foster City, CA, USA). The newly identified sequences of Rspe_dsx,Ntak_dsx, and Hsjo_dsx were deposited in the GenBank/EMBL/DDBJ database under accession numbers: LC635717, LC635718, and LC635719 (Table 1).

RNA-seq analysis in Reticulitermes speratus. RNA-seq data of R. speratus were used to compare the expression levels of Rspe_dsx (Table 1) among three castes (workers, soldiers, and reproductives), two body parts (heads and the remaining parts), and two sexes (biological triplicates; NCBI BioProject Accession No. PRJDB5589 ${ }^{21}$ ). The filtered RNA-seq reads were mapped onto their genome assembly ${ }^{20}$ using TopHat v2.1.021. Transcript abundances were then estimated using the featureCounts program of the Subread package ${ }^{49}$. To compare gene expression levels among castes and between sexes, first, counts per million (CPM) were calculated from the estimated transcript abundances. Genes with at least CPM of 1 in at least three samples were kept for subsequent analyses. CPM values were then normalized with the trimmed mean of M-values (TMM) algorithm in edge $\mathrm{R}^{50}$. Differential gene expression analyses were performed separately for each body part using a GLM with two factors, namely, caste and sex implemented in edgeR, and then, genes with a false discovery rate $($ FDR $)<0.01$ were identified as genes expressed in a sex-specific manner. RPKM (Reads Per Kilobase Million) values were calculated by dividing the CPM values by the length of the genes in kilobases.

Real-time quantitative PCR. Gene-specific primers were designed against Rspe_dsx, Ntak_dsx, and CPun_ds $x$ using Primer3Plus ${ }^{51}$ for real-time quantitative PCR (Table S2). Total RNA of female and male nymphs of $R$. speratus (from the colony collected in 2019) was individually extracted from whole bodies (10 individuals in each sex) using ISOGEN II. Total RNA of male (minor and medium) workers, female (medium and major) workers, and male soldiers of $N$. takasagoensis (from the colony collected in 2018) was extracted from the whole body of five separate individuals using ISOGEN II. Total RNAs derived from the gonads of adult woodroaches were also used. DNase treatment was performed using the same method described above. According to previous studies $^{47,48}$, incipient colonies (queen-king and two-queen colonies) of $R$. speratus were established using alates derived from three colonies collected in 2019. Eggs produced sexually in queen-king colonies and asexually in queen-queen colonies develop into both sexes and only females, respectively. Total RNA was extracted from 30 sexual and 30 asexual eggs (triplicated) and treated with DNase I using ReliaPrep RNA Tissue Miniprep System (Promega). cDNAs were synthesised from the purified RNA using a High-Capacity cDNA Reverse Transcription Kit (Applied Biosystems). Biological replications $(n=3-5)$ were prepared for each category. Expression analyses were performed using Thunderbird SYBR qPCR Mix (Toyobo, Japan) with a MiniOpticon Real-time PCR system (Bio-Rad, Japan) and an Applied Biosystems 7500 Fast Real-Time PCR System (Applied Biosystems).

To determine a sustainable internal control gene for R. speratus, the expression levels of six genes [EF1- $\alpha$ (accession no. AB602838), NADH-dh (no. AB602837), $\beta$-actin (no. AB520714), GstD1 (gene id RS001168), EIF-1 (RS005199), and RPS18 (RS015150)] were evaluated using the GeNorm ${ }^{52}$ and NormFinder ${ }^{53}$ software (Table S4). For C. punctulatus and N. takasagoensis, according to previous studies ${ }^{41,54}$, three genes [ $\beta$-actin (nos. Cp_TR19468 and AB501107), NADH-dh (Cp_TR49774 and AB50119), and EF1- $\alpha$ (AFK49795 and AB501108)] were evaluated (Tables S5 and S6). All gene-specific primers were designed using Primer3Plus (Table S2). We confirmed the amplification of a single PCR product using dissociation curves and product sizes.

The expression levels were statistically analysed using GLMs with gamma error distribution and log link function. The fixed effects were sex, caste, and their interaction. Multiple comparisons were performed using

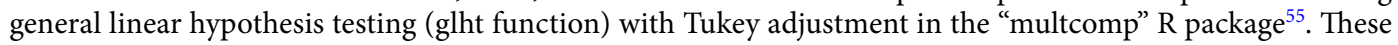
analyses were conducted using R ver. 4.0.3 (available at http://cran.r-project.org/). 
Search for transcription factor binding motif. The Reticulitermes genome was loaded into the database of HOMER v4.11 using the "loagGenome.pl" program. The "findMotifsGenome.pl" program was executed to search for Drosophila motif collections using the "-mcheck" option against the promoter of Rspe_dsx, which was the $1.0 \mathrm{kbp}$ upstream region from its transcription start site. The nucleotide sequences of Drosophila transcription factors that were detected in their binding sites were subjected to a BlastX search for orthologues against the protein database of $R$. speratus ${ }^{41}$ using BLAST $+(2.10 .1)^{56}$. The expression levels of these orthologues were extracted from their transcriptome data ${ }^{21}$ and statistically analysed using GLMs with gamma error distribution and $\log$ link function, as mentioned above.

Received: 26 May 2021; Accepted: 20 July 2021

Published online: 06 August 2021

\section{References}

1. Verhulst, E. C., van de Zande, L. \& Beukeboom, L. W. Insect sex determination: It all evolves around transformer. Curr. Opin. Genet. Dev. 20, 376-383. https://doi.org/10.1016/j.gde.2010.05.001 (2010).

2. Erickson, J. W. \& Quintero, J. J. Indirect effects of ploidy suggest X chromosome dose, not the X: A ratio, signals sex in Drosophila. PLoS Biol. 5, e332. https://doi.org/10.1371/journal.pbio.0050332 (2007).

3. Gempe, T. \& Beye, M. Function and evolution of sex determination mechanisms, genes and pathways in insects. BioEssays 33, 52-60. https://doi.org/10.1002/bies.201000043 (2011).

4. Verhulst, E. C. \& van de Zande, L. Double nexus-Doublesex is the connecting element in sex determination. Brief. Funct. Genomics 14, 396-406. https://doi.org/10.1093/bfgp/elv005 (2015).

5. Kato, Y., Kobayashi, K., Watanabe, H. \& Iguchi, T. Environmental sex determination in the branchiopod crustacean Daphnia magna: Deep conservation of a doublesex gene in the sex-determining pathway. PLoS Genet. 7, e1001345. https://doi.org/10.1371/ journal.pgen.1001345 (2011).

6. Price, D. C., Egizi, A. \& Fonseca, D. M. The ubiquity and ancestry of insect doublesex. Sci. Rep. 5, 13068. https://doi.org/10.1038/ srep13068 (2015).

7. Pomerantz, A. F. \& Hoy, M. A. Expression analysis of Drosophila doublesex, transformer-2, intersex, fruitless-like, and vitellogenin homologs in the parahaploid predator Metaseiulus occidentalis (Chelicerata: Acari: Phytoseiidae). Exp. Appl. Acarol. 65, 1-16. https://doi.org/10.1007/s10493-014-9855-2 (2015).

8. Guo, L. et al. Identification and characterization of doublesex in Bemisia tabaci. Insect Mol. Biol. 27, 620-632. https://doi.org/10. $1111 /$ imb.12494 (2018).

9. Wexler, J. et al. Hemimetabolous insects elucidate the origin of sexual development via alternative splicing. Elife 8, e47490. https:// doi.org/10.7554/eLife.47490 (2019).

10. Kopp, A. Dmrt genes in the development and evolution of sexual dimorphism. Trends Genet. 28, 175-184. https://doi.org/10. 1016/j.tig.2012.02.002 (2012).

11. Bellefroid, E. J. et al. Expanding roles for the evolutionarily conserved Dmrt sex transcriptional regulators during embryogenesis. Cell. Mol. Life Sci. 70, 3829-3845. https://doi.org/10.1007/s00018-013-1288-2 (2013).

12. An, W., Cho, S., Ishii, H. \& Wensink, P. C. Sex-specific and non-sex-specific oligomerization domains in both of the doublesex transcription factors from Drosophila melanogaster. Mol. Cell. Biol. 16, 3106-3111. https://doi.org/10.1128/mcb.16.6.3106 (1996).

13. Inward, D., Beccaloni, G. \& Eggleton, P. Death of an order: A comprehensive molecular phylogenetic study confirms that termites are eusocial cockroaches. Biol. Lett. 3, 331-335. https://doi.org/10.1098/rsbl.2007.0102 (2007).

14. Lo, N. et al. Evidence from multiple gene sequences indicates that termites evolved from wood-feeding cockroaches. Curr. Biol. 10, 801-804. https://doi.org/10.1016/s0960-9822(00)00561-3 (2000).

15. Harrison, M. C. et al. Hemimetabolous genomes reveal molecular basis of termite eusociality. Nat. Ecol. Evol. 2, 557-566. https:// doi.org/10.1038/s41559-017-0459-1 (2018).

16. Hayashi, Y. et al. Construction and characterization of normalized cDNA libraries by 454 pyrosequencing and estimation of DNA methylation levels in three distantly related termite species. PLoS ONE 8, e76678. https://doi.org/10.1371/journal.pone.0076678 (2013).

17. Mitaka, Y., Kobayashi, K. \& Matsuura, K. Caste-, sex-, and age-dependent expression of immune-related genes in a Japanese subterranean termite, Reticulitermes speratus. PLOS ONE 12, e0175417. https://doi.org/10.1371/journal.pone.0175417 (2017).

18. Poulsen, M. et al. Complementary symbiont contributions to plant decomposition in a fungus-farming termite. Proc. Natl. Acad. Sci. 111, 14500-14505. https://doi.org/10.1073/pnas.1319718111 (2014).

19. Terrapon, N. et al. Molecular traces of alternative social organization in a termite genome. Nat. Commun. 5, 3636. https://doi.org/ $10.1038 /$ ncomms4636 (2014).

20. Shigenobu, S. Reticulitermes speratus genome assembly. figshare. https://doi.org/10.6084/m9.figshare.14267342.v1 (2021).

21. Shigenobu, S. R. speratus caste RNA-seq. figshare. https://doi.org/10.6084/m9.figshare.14267498.v1 (2021).

22. Itakura, S., Yoshikawa, Y., Togami, Y. \& Umezawa, K. Draft genome sequence of the termite, Coptotermes formosanus: Genetic insights into the pyruvate dehydrogenase complex of the termite. J. Asia-Pac. Entomol. 23, 666-674. https://doi.org/10.1016/j. aspen.2020.05.004 (2020).

23. Zhang, X., Rayner, J. G., Blaxter, M. \& Bailey, N. W. Rapid parallel adaptation despite gene flow in silent crickets. Nat. Commun. 12, 50. https://doi.org/10.1038/s41467-020-20263-4 (2021).

24. Shigenobu, S. Reticulitermes speratus gene models OGS1.0. figshare. https://doi.org/10.6084/m9.figshare.14267381.v1 (2021).

25. Shigenobu, S. Macrotermes natalensis gene models MnGM3. figshare. https://doi.org/10.6084/m9.figshare.14370266.v1 (2021).

26. Baral, S., Arumugam, G., Deshmukh, R. \& Kunte, K. Genetic architecture and sex-specific selection govern modular, male-biased evolution of doublesex. Sci. Adv. 5, eaau3753. https://doi.org/10.1126/sciadv.aau3753 (2019).

27. Toyota, K. et al. Molecular cloning of doublesex genes of four cladocera (water flea) species. BMC Genomics 14, 239. https://doi. org/10.1186/1471-2164-14-239 (2013).

28. Heinz, S. et al. Simple combinations of lineage-determining transcription factors prime cis-regulatory elements required for macrophage and B cell identities. Mol. Cell 38, 576-589. https://doi.org/10.1016/j.molcel.2010.05.004 (2010).

29. Zheng, J. et al. Identification and functional analysis of the doublesex gene in the redclaw crayfish, Cherax quadricarinatus. Gene Expr. Patterns 37, 119129. https://doi.org/10.1016/j.gep.2020.119129 (2020).

30. Li, S., Li, F., Yu, K. \& Xiang, J. Identification and characterization of a doublesex gene which regulates the expression of insulin-like androgenic gland hormone in Fenneropenaeus chinensis. Gene 649, 1-7. https://doi.org/10.1016/j.gene.2018.01.043 (2018).

31. Bucek, A. et al. Evolution of termite symbiosis informed by transcriptome-based phylogenies. Curr. Biol. 29, 3728-3734.e3724. https://doi.org/10.1016/j.cub.2019.08.076 (2019). 
32. Evangelista, D. A. et al. An integrative phylogenomic approach illuminates the evolutionary history of cockroaches and termites (Blattodea). Proc. R. Soc. B Biol. Sci. 286, 20182076. https://doi.org/10.1098/rspb.2018.2076 (2019).

33. Zhu, L. et al. Sexual dimorphism in diverse metazoans is regulated by a novel class of intertwined zinc fingers. Genes Dev. 14, 1750-1764 (2000).

34. Jia, L.-Y. et al. Doublesex evolution is correlated with social complexity in ants. Genome Biol. Evol. 10, 3230-3242. https://doi.org/ 10.1093/gbe/evy250 (2018).

35. Nalepa, C. A. Altricial development in wood-feeding cockroaches: The key antecedent of termite eusociality. In Biology of Termites: A Modern Synthesis (eds Bignell, D. E. et al.) 69-96 (Springer, 2011).

36. Bourguignon, T., Hayashi, Y. \& Miura, T. Skewed soldier sex ratio in termites: Testing the size-threshold hypothesis. Insectes Soc. 59, 557-563. https://doi.org/10.1007/s00040-012-0252-8 (2012).

37. Toga, K. et al. Sexual difference in juvenile-hormone titer in workers leads to sex-biased soldier differentiation in termites. J. Insect Physiol. 87, 63-70. https://doi.org/10.1016/j.jinsphys.2016.02.005 (2016).

38. Watanabe, D., Gotoh, H., Miura, T. \& Maekawa, K. Social interactions affecting caste development through physiological actions in termites. Front. Physiol. https://doi.org/10.3389/fphys.2014.00127 (2014).

39. Oguchi, K., Maekawa, K. \& Miura, T. Regulatory mechanisms underlying the differentiation of neotenic reproductives in termites: Partial release from arrested development. Front. Ecol. Evol. https://doi.org/10.3389/fevo.2021.635552 (2021).

40. Korb, J. Genes underlying reproductive division of labor in termites, with comparisons to social hymenoptera. Front. Ecol. Evol. https://doi.org/10.3389/fevo.2016.00045 (2016).

41. Masuoka, Y., Toga, K., Nalepa, C. A. \& Maekawa, K. A crucial caste regulation gene detected by comparing termites and sister group cockroaches. Genetics 209, 1225-1234. https://doi.org/10.1534/genetics.118.301038 (2018).

42. Priyam, A. et al. Sequenceserver: A modern graphical user interface for custom BLAST databases. Mol. Biol. Evol. 36, $2922-2924$. https://doi.org/10.1093/molbev/msz185 (2019).

43. Kato, Y. et al. Molecular cloning and sexually dimorphic expression of DM-domain genes in Daphnia magna. Genomics 91, 94-101. https://doi.org/10.1016/j.ygeno.2007.09.002 (2008).

44. Oliveira, D. C. S. G. et al. Identification and characterization of the doublesex gene of Nasonia. Insect Mol. Biol. 18, 315-324. https:// doi.org/10.1111/j.1365-2583.2009.00874.x (2009).

45. Kumar, S., Stecher, G. \& Tamura, K. MEGA7: Molecular evolutionary genetics analysis version 7.0 for bigger datasets. Mol. Biol. Evol. 33, 1870-1874. https://doi.org/10.1093/molbev/msw054 (2016).

46. Ronquist, F. \& Huelsenbeck, J. P. MrBayes 3: Bayesian phylogenetic inference under mixed models. Bioinformatics 19, 1572-1574. https://doi.org/10.1093/bioinformatics/btg180 (2003).

47. Maekawa, K., Ishitani, K., Gotoh, H., Cornette, R. \& Miura, T. Juvenile Hormone titre and vitellogenin gene expression related to ovarian development in primary reproductives compared with nymphs and nymphoid reproductives of the termite Reticulitermes speratus. Physiol. Entomol. 35, 52-58. https://doi.org/10.1111/j.1365-3032.2009.00711.x (2010).

48. Miyazaki, S. et al. Intracolonial genetic variation affects reproductive skew and colony productivity during colony foundation in a parthenogenetic termite. BMC Evol. Biol. 14, 177. https://doi.org/10.1186/s12862-014-0177-0 (2014).

49. Liao, Y., Smyth, G. K. \& Shi, W. featureCounts: An efficient general purpose program for assigning sequence reads to genomic features. Bioinformatics 30, 923-930. https://doi.org/10.1093/bioinformatics/btt656 (2013).

50. Robinson, M. D., McCarthy, D. J. \& Smyth, G. K. edgeR: A Bioconductor package for differential expression analysis of digital gene expression data. Bioinformatics 26, 139-140. https://doi.org/10.1093/bioinformatics/btp616 (2009).

51. Untergasser, A. et al. Primer3Plus, an enhanced web interface to Primer3. Nucleic Acids Res. 35, W71-W74. https://doi.org/10. 1093/nar/gkm306 (2007).

52. Vandesompele, J. et al. Accurate normalization of real-time quantitative RT-PCR data by geometric averaging of multiple internal control genes. Genome Biol. 3, research0034.0031. https://doi.org/10.1186/gb-2002-3-7-research0034 (2002).

53. Andersen, C. L., Jensen, J. L. \& Ørntoft, T. F. Normalization of real-time quantitative reverse transcription-PCR data: A modelbased variance estimation approach to identify genes suited for normalization, applied to bladder and colon cancer data sets. Can. Res. 64, 5245-5250. https://doi.org/10.1158/0008-5472.can-04-0496 (2004).

54. Toga, K., Hojo, M., Miura, T. \& Maekawa, K. Expression and function of a limb-patterning gene Distal-less in the soldier-specific morphogenesis in the nasute termite Nasutitermes takasagoensis. Evol. Dev. 14, 286-295. https://doi.org/10.1111/j.1525-142X. 2012.00545.x (2012).

55. Hothorn, T., Bretz, F. \& Westfall, P. Simultaneous inference in general parametric models. Biom. J. 50, 346-363. https://doi.org/ 10.1002/bimj.200810425 (2008).

56. Camacho, C. et al. BLAST+: Architecture and applications. BMC Bioinform. 10, 421. https://doi.org/10.1186/1471-2105-10-421 (2009).

57. Bourguignon, T. et al. Transoceanic dispersal and plate tectonics shaped global cockroach distributions: Evidence from mitochondrial phylogenomics. Mol. Biol. Evol. 35, 970-983. https://doi.org/10.1093/molbev/msy013 (2018).

\section{Acknowledgements}

Christine A. Nalepa kindly provided us with Cryptocercus punctulatus and gave us valuable comments to the final version of our manuscript. Shutaro Hanmoto provided assistance with laboratory work. We thank Toru Miura, Masatoshi Matsunami, Dominic Evangelista, and an anonymous reviewer for their constructive suggestions regarding this study, and Functional Genomics Facility, NIBB Core Research Facilities for technical support. This study was partly supported by JSPS KAKENHI (Grant Numbers JP19K06860 to SM, JP20K06816 to YH, and JP19H03273 to KM) and by the NIBB Collaborative Research Program (Nos. 19-335 and 20-323).

\section{Author contributions}

S.M., H.G., and K.M. planned and designed the experiments. S.M., K.F., K.K., and Y.M. collected the samples. S.M., H.G., T.N., Y.H., and S.S. searched for homologues. S.M., K.F. and K.M. contributed to the phylogenetic analysis. S.M., K.F., K.K., Y.M., and Y.H. performed the gene expression analyses. S.M. and S.S. analysed the data. S.M., K.F., T.N., and K.M. drafted the manuscript. All authors read and approved the final manuscript.

\section{Competing interests}

The authors declare no competing interests.

\section{Additional information}

Supplementary Information The online version contains supplementary material available at https://doi.org/ 10.1038/s41598-021-95423-7. 
Correspondence and requests for materials should be addressed to S.M. or K.M.

Reprints and permissions information is available at www.nature.com/reprints.

Publisher's note Springer Nature remains neutral with regard to jurisdictional claims in published maps and institutional affiliations.

(c) (i) Open Access This article is licensed under a Creative Commons Attribution 4.0 International cc) License, which permits use, sharing, adaptation, distribution and reproduction in any medium or format, as long as you give appropriate credit to the original author(s) and the source, provide a link to the Creative Commons licence, and indicate if changes were made. The images or other third party material in this article are included in the article's Creative Commons licence, unless indicated otherwise in a credit line to the material. If material is not included in the article's Creative Commons licence and your intended use is not permitted by statutory regulation or exceeds the permitted use, you will need to obtain permission directly from the copyright holder. To view a copy of this licence, visit http://creativecommons.org/licenses/by/4.0/.

(C) The Author(s) 2021, corrected publication 2021 